\title{
Muon $g-2$ anomaly confronted with the higgs global data in the left-right twin Higgs models
}

\author{
Guo-Li Liu ${ }^{1, a}$, Qing-Guo Zeng ${ }^{2, b}$ \\ ${ }^{1}$ School of Physics, Zhengzhou University, Zhengzhou 450000, People's Republic of China \\ ${ }^{2}$ School of Physics, Shangqiu Normal University, Shangqiu 470000, People's Republic of China
}

Received: 2 April 2019 / Accepted: 29 May 2019 / Published online: 19 July 2019

(C) The Author(s) 2019

\begin{abstract}
We will examine the muon $g-2$ anomaly with the background of the Higgs global fit data in the framework of the Left-Right Twin Higgs (LRTH) Models. The joint constrains of the precision electroweak data, the 125 $\mathrm{GeV}$ Higgs data, the leptonic flavor changing decay $\mu \rightarrow e \gamma$ decays, and the mass requirement of the right-handed neutrino $v_{R}$, the vector-like top partner $T$ and the heavy gauge boson $W_{H}, m_{v_{R}}>m_{T}>m_{W_{H}}$, are all considered in our calculation. Furthermore, since the neutral scalar $\phi^{0}$ may be lighter than the $125 \mathrm{GeV}$ Higgs, the direct searches from the $h \rightarrow \phi^{0} \phi^{0}$ channels can impose stringent upper limits on $\operatorname{Br}\left(h \rightarrow \phi^{0} \phi^{0}\right)$, which will reduce the allowed region of $m_{\phi^{0}}$ and $f$, the vacuum expectation value of the SM right-handed Higgs $H_{R}$. It is concluded that the muon g-2 anomaly can be explained in the region of $700 \mathrm{GeV} \leq f \leq 1100 \mathrm{GeV}, 13$ $\mathrm{GeV} \leq m_{\phi^{0}} \leq 55 \mathrm{GeV}, 100 \mathrm{GeV} \leq m_{\phi^{ \pm}} \leq 900 \mathrm{GeV}, m_{v_{R}} \geq$ $15 \mathrm{TeV}$, and $200 \mathrm{GeV} \leq M \leq 800 \mathrm{GeV}$, after imposing all the constraints mentioned above, where $M$ here means the mass mixing coefficient $M \bar{q}_{L} q_{R}$, allowed by gauge invariance.
\end{abstract}

\section{Introduction}

The muon anomalous magnetic moment $(g-2)$ is a very precisely measured observable, and expected to shed light on new physics. The muon $g-2$ anomaly has been a long-standing puzzle since the announcement by the E821 experiment in 2001 [1,2]. The precision measurement of $a_{\mu}=(g-2) / 2$ has been performed by the E821 experiment at Brookhaven National Laboratory [3], with the current world-averaged result given by [4]

$a_{\mu}^{\exp }=116,592,091( \pm 54)( \pm 33) \times 10^{-11}$.

\footnotetext{
a e-mail: guoliliu@zzu.edu.cn

b e-mail: zengqingguo66@126.com
}

Meanwhile, the Standard Model (SM) prediction from the Particle Data Group gives [4],

$a_{\mu}^{S M}=116,591,803( \pm 1)( \pm 42)( \pm 26) \times 10^{-11}$.

The difference between experiment and theory is

$\Delta a_{\mu}=a_{\mu}^{\exp }-a_{\mu}^{S M}=(288 \pm 80) \times 10^{-11}$,

which shows a $3.6 \sigma$ discrepancy, hinting at tantalizing new physics beyond the SM. It is the difference between the experimental data and the SM prediction determines the room for new physics.

There exist various new physics scenarios to explain the muon $g-2$ excess, for recent reviews, see e.g. Refs. [5-9]. Among these extensions, the LRTH model may also provide a explanation for the muon $g-2$ anomaly. In these models, there are six massive gauge bosons left after the symmetry breaking: the $\mathrm{SM} Z$ and $W^{ \pm}$, and extra heavier bosons, $Z_{H}$ and $W_{H}^{ \pm}$. And these models also include eight scalars: one neutral pseudoscalar, $\phi^{0}$, a pair of charged scalars $\phi^{ \pm}$, the SM physical Higgs $h$, and an $\mathrm{SU}(2)_{L}$ twin Higgs doublet $\hat{h}=\left(\hat{h}_{1}^{+}, \hat{h}_{2}^{0}\right)$. The lepton couplings to the pseudoscalar can be sizably enhanced by the large right-handed neutrino mass $m_{v_{R}}$. The pseudoscalar can give positive contributions to muon $g-2$ via the two-loop Barr-Zee diagrams.

In this work we will examine the parameter space of LRTH by considering the joint constraints from the theory, the precision electroweak data, the $125 \mathrm{GeV}$ Higgs signal data, the muon $g-2$ anomaly, the lepton rare decay of $\mu \rightarrow e \gamma$, as well as the direct search limits from the LHC.

This work is organized as follows. In Sect. 2 we recapitulate the effective couplings of the LRTH model. In Sect. 3 the muon $g-2$ anomaly and other relevant constraints are discussed. Using the direct search limits from the LHC, especially the Higgs global fit to constrain the model is the topic of the Sect. 4. Finally, Sect. 6 comes to the conclusion. 


\section{The relevant couplings in the LRTH models}

To implement the twin Higgs mechanism, a global symmetry which is partially gauged and spontaneously broken, is needed. At the same time, to control the quadratic divergences, the twin symmetry, which is identified with the left-right symmetry interchanging $L$ and $R$, is also necessary. The left-right symmetry implies that, for the gauge couplings $g_{2 L}$ and $g_{2 R}$ of $S U(2)_{L}$ and $S U(2)_{R}$, $g_{2 L}=g_{2 R}=g_{2}$.

In the LRTH model proposed in [10-19], the global symmetry can be chosen as $U(4) \times U(4)$ and the gauge subgroup $S U(2)_{L} \times S U(2)_{R} \times U(1)_{B-L}$. With the global symmetry, the Higgs field and the twin Higgs in the fundamental representation of each $U(4)$ can be written as $H=\left(H_{L}, H_{R}\right)$ and $\hat{H}=\left(\hat{H}_{L}, \hat{H}_{R}\right)$, respectively. After each Higgs develops a vacuum expectation value (VEV),

$\langle H\rangle^{T}=(0,0,0, f), \quad\langle\hat{H}\rangle^{T}=(0,0,0, \hat{f})$,

the global symmetry $U(4) \times U(4)$ breaks to $U(3) \times U(3)$, with the gauge group $S U(2)_{L} \times S U(2)_{R} \times U(1)_{B-L}$ down to the $\operatorname{SM} U(1)_{Y}$.

After the Higgses obtain VEVs as shown in Eq. (4), the breaking of the $S U(2)_{L} \times S U(2)_{R} \times U(1)_{B-L}$ to $S U(2)_{L} \times U(1)_{B-L}$ generates three new massive gauge bosons, with masses proportional to $\sqrt{f^{2}+\hat{f}^{2}}$. The couplings of the these gauge bosons to the SM particles, greatly constrain their masses. The masses of the these extra gauge bosons can be large enough to avoid the constraints from the electroweak precision measurements by requiring $\hat{f} \gg f$. Furthermore, the problems induced by the large value of $\hat{f}$, can be eliminated by imposing certain discrete symmetry which requires that the $\hat{H}$ is odd while all the other fields are even so as to ensure the Higgs field $\hat{H}$ couples only to the gauge sector as described in Ref. [17].

In such models, with the global symmetry breaking from $\mathrm{U}(4) \times \mathrm{U}(4)$ to $\mathrm{U}(3) \times \mathrm{U}(3)$, fourteen Goldstone bosons are generated, six of which are eaten by the massive gauge bosons $Z_{H}$ and $W_{H}^{ \pm}$and the SM gauge bosons $Z^{0}$ and $W^{ \pm}$, while the rest of the Goldstone bosons consist of the scalar fields: one neutral pseudoscalar, $\phi^{0}$, a pair of charged scalars $\phi^{ \pm}$, the SM physical Higgs $h$, and an $\mathrm{SU}(2)_{L}$ twin Higgs doublet $\hat{h}=\left(\hat{h}_{1}^{+}, \hat{h}_{2}^{0}\right)$.

Since the effective Yukawa couplings is suppressed by $f / \Lambda$, with $\Lambda=4 \pi \hat{f}$, iti is difficult to account for the $\mathcal{O}(1)$ top Yukawa coupling. To give the large top quark mass, vector-like quarks are introduced. The vector-like top quark also cancels the leading quadratic divergence of the SM gauge boson mass contributed by the top quark in the loop level. so the hierarchy problem induced by the top quark are settled down. ${ }^{1}$ At the same time, the new particles such as the gauge bosons and the vector-like top singlet in the LRTH models have rich phenomenology at the LHC (see for examples [17-19,23-29]).

Based on the Lagrangian given in Ref. [17], the fermion couplings that are related to our calculation can be written out in Table 1,

where the mixing angles are [17]

$$
\begin{aligned}
S_{L} & \sim \sin \alpha_{L} \sim \frac{M}{m_{T}} \sin x, S_{R} \sim \sin \alpha_{R} \sim \frac{M}{m_{T}}(1+\sin 2 x), \\
x & =\frac{v}{\sqrt{2} f} .
\end{aligned}
$$

As for the parameter $M$ above, as we know, in the gauge invariant top Yukawa terms, there is the mass mixing term $M \bar{q}_{L} q_{R}$, allowed by gauge invariance. $M \neq 0$ means there is mixing between the SM-like top quark and the heavy top quark. The mixing parameter $M$ also be constrained by the $Z \rightarrow \bar{b} b$ branching ratio and oblique parameters and it usually prefers to a small value $[17,30]$, but the constraint is not too strong, and we can loose it.

Neutrino oscillations (see for example [31-34]) imply that neutrinos are massive, and the LRTH models try to explain the origin of the neutrino masses and mass hierarchy $[18,19]$. To provide lepton masses in the LRTH models, one can introduce three families doublets $\mathrm{SU}(2)_{L, R}$ which are charged under $S U(3)_{c} \times S U(2)_{L} \times S U(2)_{R} \times U(1)_{B-L}$ as

$L_{L \alpha}=-i\left(\begin{array}{c}v_{L \alpha} \\ l_{L \alpha}\end{array}\right):(\mathbf{1}, \mathbf{2}, \mathbf{1},-1)$,

$L_{R \alpha}=\left(\begin{array}{c}v_{R \alpha} \\ l_{R \alpha}\end{array}\right):(\mathbf{1}, \mathbf{1}, \mathbf{2},-1)$,

where the family index $\alpha$ runs from 1 to 3 .

Leptons can acquire masses via non-renormalisable dimension 5 operators. The charged leptons obtain their masses via the following non-renormalisable dimension 5 operators,

$\frac{y_{l}^{i j}}{\Lambda}\left(\bar{L}_{L i} H_{L}\right)\left(H_{R}^{\dagger} L_{R j}\right)+\frac{y_{\nu}^{i j}}{\Lambda}\left(\bar{L}_{L, i} \tau_{2} H_{L}^{*}\right)\left(H_{R}^{T} \tau_{2} L_{R j}\right)+$ H.c.,

which will give rise to lepton Dirac mass terms $y_{v, l}^{i j} v f / \Lambda$, once $H_{L}$ and $H_{R}$ acquire VEVs.

The Majorana nature of the left- and right-handed neutrinos, however, makes one to induce Majorana terms ( only the mass section) in dimension 5 operators,

$\frac{c_{L}}{\Lambda}\left(\bar{L}_{L \alpha} \tau_{2} H_{L}^{\dagger}\right)^{2}+$ H.c, $\quad \frac{c_{R}}{\Lambda}\left(\bar{L}_{R \alpha} \tau_{2} H_{R}^{\dagger}\right)^{2}+$ H.c.

\footnotetext{
1 The leading quadratically divergent of the Higgs masses contributed by the SM gauge boson are canceled by the loop involving the heavy gauge bosons.
} 
Table 1 The three-point couplings of the charged gauge boson-fermion-fermion and those of the scalar-fermion-fermion in the LRTH models. The chirality projection operators are $P_{R, L}=\left(1 \pm \gamma_{5}\right) / 2$

\begin{tabular}{llll}
\hline Particles & Vertexes & Particles & Vertexes \\
\hline$W_{H \mu}^{+} \bar{t} b$ & $e \gamma_{\mu} S_{R} P_{R} /\left(\sqrt{2} s_{w}\right)$ & $W_{H}^{+\mu} \bar{T} b$ & $e \gamma_{\mu} C_{R} P_{L} /\left(\sqrt{2} s_{w}\right)$ \\
$\Phi^{+} \bar{t} b$ & $-i\left(S_{R} m_{b} P_{L}-y S_{L} f P_{R}\right) / f$ & $\Phi^{+} \bar{T} b$ & $i\left(C_{R} m_{b} P_{L}-y C_{L} f P_{R}\right) / f$ \\
\hline
\end{tabular}

Once $H_{L}\left(H_{R}\right)$ obtains a VEV, both neutrino chiralities obtain Majorana masses via these operators, the smallness of the light neutrino masses, however, can not be well explained.

However, if we assume that the twin Higgs $\hat{H}_{R}$ is forbidden to couple to the quarks to prevent the heavy top quark from acquiring a large mass of order $y \hat{f}$, but it can couple to the right-handed neutrinos, one may find that $[18,19]$

$\frac{c_{\hat{H}}}{\Lambda}\left(\bar{L}_{R \alpha} \tau_{2} \hat{H}_{R}^{\dagger}\right)^{2}+$ H.c.,

which will give a contribution to the Majorana mass of the heavy right-handed neutrino $v_{R}$, in addition to those of Eq. (7).

So after the electroweak symmetry breaking, $H_{R}$ and $\hat{H}_{R}$ get VEVs, $f$ and $\hat{f}$ (Eq. (4)), respectively, we can derive the following seesaw mass matrix for the LRTH model in the basis $\left(v_{L}, v_{R}\right)$ :

$\mathcal{M}=\left(\begin{array}{cc}c \frac{v^{2}}{2 \Lambda} & y_{v} \frac{v f}{\sqrt{2} \Lambda} \\ y_{v}^{T} \frac{v f}{\sqrt{2} \Lambda} & c \frac{f^{2}}{\Lambda}+c_{\hat{H}} \frac{\hat{f}^{2}}{\Lambda}\end{array}\right)$.

In the one-generation case there is two massive states, a heavy $\left(\sim v_{R}\right)$ and a light one. For the case that $v<f<\hat{f}$, the masses of the two eigenstates are about $m_{v_{\text {heavy }}} \sim c_{\hat{H}} \frac{\hat{f}^{2}}{\Lambda}$ and $m_{v_{\text {light }}}=\frac{c v^{2}}{2 \Lambda}[18,19]$.

The Lagrangian in Eqs. (6)-(8) induces neutrino masses and the mixings of different generation leptons, which may be a source of lepton flavour violating $[18,19]$. In our case we will consider the contributions to the lepton flavour violating of the charged scalars, $\phi^{ \pm}$and the heavy gauge boson, $W_{H}$. The relevant vertex interactions for these processes are explicated in the followings:

$\phi^{-} \bar{l} \nu_{L, R}: \frac{i}{f}\left(m_{l_{L}, v_{R}} P_{L}-m_{v_{L}, l_{R}} P_{R}\right) V_{H} \sim i c_{H} \frac{\hat{f}^{2}}{\Lambda f} V_{H} P_{L}$,

$W_{L, R}^{-} \bar{l} v_{L, R}: \frac{e}{\sqrt{2} s_{w}} \gamma_{\mu} P_{L, R} V_{H}$

where $V_{H}$ is the mixing matrix of the heavy neutrino and the leptons mediated by the charged scalars and the heavy gauge bosons. The vertexes of $\phi^{-} \bar{l} \nu_{L, R}$ can also be expressed in the coupling constants. The $\phi^{-} \bar{l} v_{R}$, for example, is also written as $i c_{H} \frac{\hat{f}^{2}}{\Lambda f} P_{L}$ if we neglect the charged lepton masses and take $m_{v_{h}}=c_{H} \hat{f}^{2} / \Lambda$.

\section{Muon $g-2$ anomaly and relevant constraints}

\subsection{Numerical calculations}

In this paper, the light CP-even Higgs $h$ is taken as the SMlike Higgs, $m_{h}=125 \mathrm{GeV}$. Since the muon $g-2$ anomaly favors a light charged pseudoscalar with a large coupling to lepton and a heavy right-handed neutrinos, we scan over $m_{\phi}$ and $m_{v_{R}}$ in the following ranges [17-19]:

$$
\begin{aligned}
100 \mathrm{GeV} & <m_{\phi^{ \pm}}<1000 \mathrm{GeV}, \quad 5000 \mathrm{GeV} \\
& <m_{v_{R}}<50000 \mathrm{GeV} .
\end{aligned}
$$

In the following calculation, the following constraints are considered:

1. From theoretical constraints and precision electroweak data, The theoretical constraints such as those from the unitarity and coupling-constant perturbativity, and the constraints from the oblique parameters $S, T, U$ will be considered [35].

2. From the lepton number violating signals of the top partners $[18,19,23]$, we can see that right-handed neutrinos prefer to have a very large mass and the charged scalars are heavy. we can also have the constrains $m_{v_{R}}>m_{T}$ and $m_{T}>m_{W_{H}}$ [23].

3. The constraints from the signal data of the $125 \mathrm{GeV}$ Higgs will be important, since the couplings of the $125 \mathrm{GeV}$ Higgs with the SM particles in LRTH model can deviate from the SM ones and the SM-like decay modes may be modified severely. Moreover, when $m_{\phi^{0}}$ is smaller than $m_{h} / 2=62.5 \mathrm{GeV}$, the decay $h \rightarrow \phi^{0} \phi^{0}$ is kinematically allowed, and the experimental data of the $125 \mathrm{GeV}$ Higgs will constrain it. We will perform $\chi_{h}^{2}$ calculation for the signal strengths of the $125 \mathrm{GeV}$ Higgs, which will be discussed detailedly in Sect. 4.

4. $f$ and $M$ parameter: The indirect constraints on $f$ come from the $Z$-pole precision measurements, the low energy neutral current process and the high energy precision measurements off the Z-pole: all these data prefer the parameter $\mathrm{f}$ to be larger than $500-600 \mathrm{GeV}$ [17]. On the other hand, it cannot be too large since the fine tuning is more severe for large $f$.

In the LRTH, furthermore, the mass of the top partner $T$ is determined by the given values of $f$ and $M$. Currently, the masses of the new heavy particles, such as 


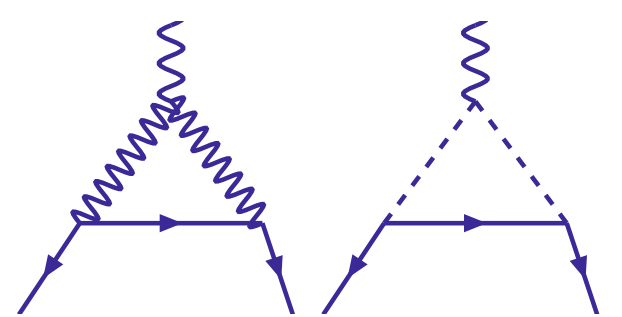

Fig. 1 The one-loop contributions to $a_{\mu}$ in the LRTH models

the $T$ have been constrained by the LHC experiments, as described in Refs. [36-44]. In other words, the LHC data also imply some indirect constraints on the allowed ranges of both the parameters $f$ and $M$ through their correlations with $m_{T}$, as discussed in Ref. [45]. For example, the top partner $T$ with mass below $656 \mathrm{GeV}$ are excluded at $95 \%$ confidence level according to the ATLAS data [46] if one takes the assumption of a branching ratio $B R\left(T \rightarrow W^{+} b\right)=1$.

By taking the above constraints from the electroweak precision measurements and the LHC data into account, we here assume that the values of the parameter $f$ and $M$ are in the ranges of

$500 \mathrm{GeV} \leq f \leq 1500 \mathrm{GeV}, \quad 0 \leq M \leq 800 \mathrm{GeV}$,

in our numerical evaluations.

5. Constraints for $f$ from the flavor changing decay $\mu \rightarrow$ $e \gamma$ : With the experimental constraints given in the first reference of $[18,19], \mathrm{BR}(\mu \rightarrow e \gamma)<1.2 \times 10^{-11}[20]^{2}$ based on the couplings in Eqs. (10) and (11), when 5\% fine-tuning is allowed, flavor changing decay $\mu \rightarrow e \gamma$ will give $f \sim[0.6-2] \mathrm{TeV}$, which basically coincide with Eq. (13).

\subsection{Muon $g-2$ in the LRTH models}

In the LRTH, the muon $g-2$ contributions are obtained via the one-loop diagrams induced by the Higgs bosons and also from the two-loop Barr-Zee diagrams mediated by $\phi^{0}, h$ and $\phi^{ \pm}$.

The one-loop contributions are give in the following, and the corresponding figures are given in Fig. 1.

We can write down them one by one [47-49]: $\Delta a_{\mu}^{\mathrm{LRTH}}(1 \text { loop })_{W}=\frac{e^{2}}{2 s_{W}^{2}} \frac{m_{\mu}^{2}}{8 \pi^{2}} \int_{0}^{1} d x \frac{-6 x^{3}-2 x^{2}}{m_{W_{H}}^{2} x+m_{v_{R}}^{2}(1-x)}$,

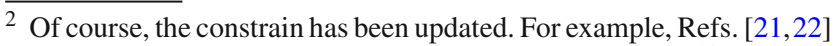
have shown the new constrain: $\operatorname{BR}(\mu \rightarrow e \gamma)<4.2 \times 10^{-13}$.
}

$$
\Delta a_{\mu}^{\mathrm{LRTH}}(1 \text { loop })_{H}=\frac{m_{v_{R}}^{2}}{f^{2}} \frac{m_{\mu}^{2}}{8 \pi^{2}} \int_{0}^{1} d x \frac{2\left(x^{3}-x^{2}\right)}{m_{H}^{2} x+m_{v_{R}}^{2}(1-x)}
$$

Before we immerse into the two-loop contribution, we discuss the coupling between the boson and the scalars. In order to go to the unitary gauge, one need know which combinations of the scalars are eaten by the massive gauge bosons $W^{ \pm}, Z$ and $W_{H}^{ \pm}, Z_{H}$. Reference [17] ask that the gaugeHiggs mixing terms from the covariant kinetic terms of $H$ and $\hat{H}$ vanish, that is, by requiring all gauge-Higgs mixing terms vanishing after the redefinition of the Higgs fields. The following is one of the re-parametrization projects corresponding to correct unitary gauge choice and is canonically normalized:

$$
\begin{array}{ll}
N \rightarrow \frac{\sqrt{2} \hat{f}}{F\left(\cos x+2 \frac{\sin x}{x}\right)} \phi^{0}, & \hat{N} \rightarrow-\frac{\sqrt{2} f \cos x}{3 F} \phi^{0}, \\
h_{1} \rightarrow 0, & h_{2} \rightarrow \frac{v+h}{\sqrt{2}}-i \frac{x \hat{f}}{\sqrt{2} F\left(\cos x+2 \frac{\sin x}{x}\right)} \phi^{0}, \\
C \rightarrow-\frac{x \hat{f}}{F \sin x} \phi^{+}, & \hat{C} \rightarrow \frac{f \cos x}{F} \phi^{+} .
\end{array}
$$

where the $N, \hat{N}, h_{1}, h_{2}, C, \hat{C}$ are in the Goldstone bosons fields, and

$$
\begin{gathered}
H=i \frac{\sin \sqrt{\chi}}{\sqrt{\chi}} e^{i \frac{N}{2 f}}\left(\begin{array}{c}
h_{1} \\
h_{2} \\
C \\
N-i f \sqrt{\chi} \cot \sqrt{\chi}
\end{array}\right), \\
\hat{H}=i \frac{\sin \sqrt{\hat{\chi}}}{\sqrt{\hat{\chi}}} e^{i \frac{\hat{N}}{2 \hat{f}}}\left(\begin{array}{c}
\hat{h}_{2} \\
\hat{C} \\
\hat{N}-i \hat{f} \sqrt{\hat{\chi}} \cot \sqrt{\hat{\chi}}
\end{array}\right) .
\end{gathered}
$$

By this parameterization, the requirement of vanishing gauge-Higgs mixing terms can be satisfied, i.e, in this redefinition of the Higgs fields, the couplings $W Z \phi^{+}, W \gamma \phi^{+}$, $W W \phi^{0}, W Z_{H} \phi^{+}, W \gamma_{H} \phi^{+}, W \phi^{0} \phi^{+}$, and $W h \phi^{+}$are zero, which has been verified and is quite different with those in other models such as the littlest Higgs models [50].

Since the coupling between the boson and the scalars $W \gamma \phi^{+}, W h \phi^{+}$and $W \phi^{0} \phi^{+}$have been vanished, so the BarrZee 2-loop diagrams (e) (f) (c) in Fig. 2 disappear. Barr-Zee 2-loop diagrams (a) (b) in Fig. 2 may not be negligible even though the vertexes such as $\phi^{0} \bar{\mu} \mu$ is very small, which is proportional to the muon mass, much smaller than the masses the top and heavy top in our special case. So there are (a) (b) (d) left contributing to $a_{\mu}$.

We can write down the Barr-Zee two-loop contributions of the diagram (a) (b) (d) respectively [51-64,76], 
Fig. 2 The potential two-loop contributions to $a_{\mu}$ the LRTH models

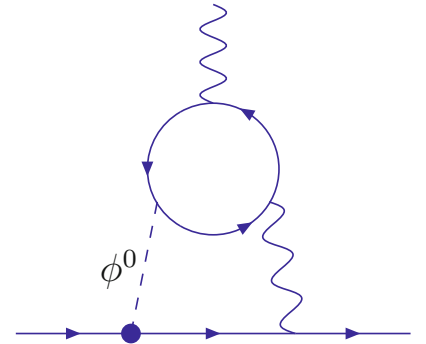

(a)

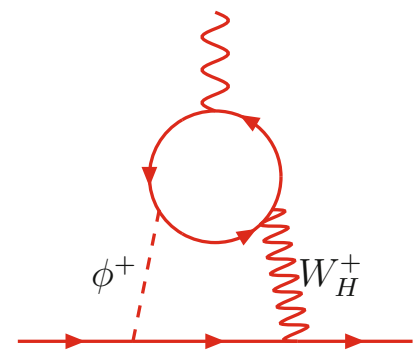

(d)

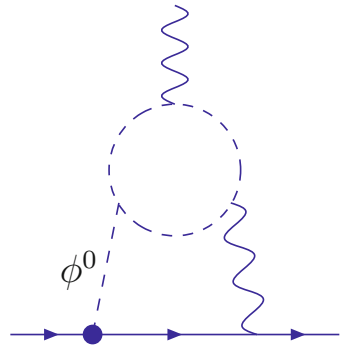

(b)

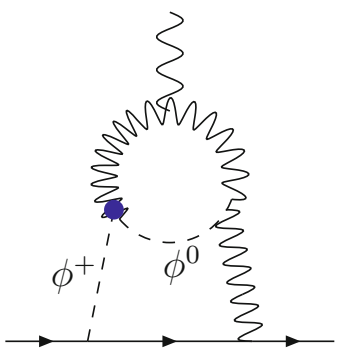

(e)

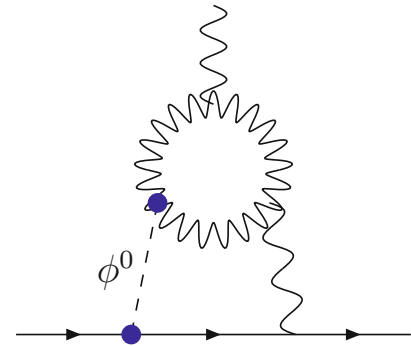

(c)

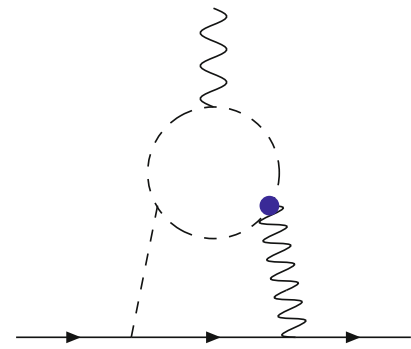

(f)

$$
\begin{aligned}
\Delta a_{\mu}^{(a)}= & -\frac{4 m_{\mu}^{2}}{e} \frac{-e^{3}}{128 \pi^{4}} \sum_{f_{j}=t, T} \frac{N_{f}^{c} Q_{f}^{2}}{m_{\mu}} \\
& \sum_{i=h, \phi^{0}} \Gamma_{\ell_{f} \ell_{i}}^{i} \Gamma_{f_{j} f_{j}}^{i} \frac{m^{f_{j}}}{m_{i}^{2}} g_{i}^{(a)}\left(r_{f_{j}}^{i}\right),
\end{aligned}
$$

where $N_{f}^{c}$ and $Q_{f}$ are the number of colours and charge of fermion $f$, respectively, $\Gamma_{f_{j} f_{i}}^{i} \mathrm{~s}$ are the couplings of the scalars to the fermions, and $r_{f}^{i} \equiv m_{f}^{2} / m_{\mu}^{2}$. The loop function is given by

$g_{i}^{(a)}(r)=\int_{0}^{1} \mathrm{~d} x \frac{N_{i}(x)}{x(1-x)-r} \ln \left(\frac{x(1-x)}{r}\right)$,

where

$$
\begin{aligned}
N_{h}(x)= & 2 x(1-x)-1, \quad N_{\phi^{0}}(x)=-1 . \\
\Delta a_{\mu}^{(b)}= & -\frac{4 m_{\mu}^{2}}{e} \frac{e^{3}}{128 \sqrt{2} \pi^{4}} \frac{v}{m_{\mu}} \\
& \sum_{i=h, \phi^{0}} \frac{\Gamma_{\ell_{f} \ell_{i}}^{i}}{m_{i}^{2}} \zeta^{i} \lambda_{H^{+} H^{-} H_{i}^{0}} g_{i}^{(b)}\left(\frac{m_{H^{+}}^{2}}{m_{i}^{2}}\right),
\end{aligned}
$$

where $\zeta^{h}=-\zeta^{H}=-\zeta^{A}=1$ and the loop function is

$$
\begin{aligned}
g_{h, H, A}^{(b)}(r)= & \int_{0}^{1} \mathrm{~d} x \frac{x(1-x)}{x(1-x)-r} \ln \left(\frac{r}{x(1-x)}\right) . \\
\Delta a_{\mu}^{(d)}(t t b+b b t)=- & \frac{4 m_{\mu}^{2}}{e} \frac{-e^{3} S_{R} V_{H}}{1024 \pi^{4} \sin ^{2} \theta_{w}} \frac{N_{t}^{c} V_{t b}^{*}}{m_{\phi}^{2}-m_{W_{H}}^{2}} \\
& \times \int_{0}^{1} d x\left[Q_{t} x+Q_{b}(1-x)\right] \\
& \times\left[G\left(\frac{m_{t}^{2}}{m_{H^{+}}^{2}}, \frac{m_{b}^{2}}{m_{H^{+}}^{2}}\right)-G\left(\frac{m_{t}^{2}}{m_{W}^{2}}, \frac{m_{b}^{2}}{m_{W}^{2}}\right)\right]
\end{aligned}
$$

$$
\begin{aligned}
& \times\left[\left(\Gamma_{t b}^{\phi^{+}, L^{*}} \Gamma_{v_{f} \mu}^{\phi^{+}}\right) \frac{m_{b}}{m_{\mu}} x(1-x)\right. \\
& \left.-\left(\Gamma_{t b}^{\phi^{+}, R^{*}} \Gamma_{v_{f}}^{\phi^{+}}\right) \frac{m_{t}}{m_{\mu}} x(1+x)\right] \\
\Delta a_{\mu}^{(d)}(T T b+b b T)=- & \frac{4 m_{\mu}^{2}}{e} \frac{-e^{3} C_{R} V_{H}}{1024 \pi^{4} \sin ^{2} \theta_{w}} \frac{1}{m_{\phi}^{2}-m_{W_{H}}^{2}} \\
& \times \int_{0}^{1} d x\left[Q_{T} x+Q_{b}(1-x)\right] \\
& \times\left[G\left(\frac{m_{T}^{2}}{m_{H}^{2}}, \frac{m_{b}^{2}}{m_{H^{+}}^{2}}\right)-G\left(\frac{m_{T}^{2}}{m_{W}^{2}}, \frac{m_{b}^{2}}{m_{W}^{2}}\right)\right] \\
& \times\left[\left(\Gamma_{T b}^{\phi^{+}, R^{*}} \Gamma_{v_{f} \mu}^{\phi^{+}}\right) \frac{m_{b}}{m_{\mu}} x(1-x)\right. \\
& \left.-\left(\Gamma_{T b}^{\phi^{+}, L^{*}} \Gamma_{v_{f} \mu}^{\phi^{+}}\right) \frac{m_{T}}{m_{\mu}} x(1+x)\right]
\end{aligned}
$$

where the loop function is defined as,

$G\left(r^{a}, r^{b}\right)=\frac{\ln \left(\frac{r^{a} x+r^{b}(1-x)}{x(1-x)}\right)}{x(1-x)-r^{a} x-r^{b}(1-x)}$,

and $\Gamma_{t b}^{\phi^{+}, R}$ and $\Gamma_{t b}^{\phi^{+}, L}$ are the right-handed and left-handed couplings of the vertex $\phi^{+} \bar{t} b$, which are given in Table 1 . From Table 1, we also see that the top vector-like partner $T$ enter into the triangle loop just as the top quark, and the contribution to $a_{\mu}$ is

$$
\begin{aligned}
& \Delta a_{\mu}^{T T b}(2 \text { loop - BZ) } \\
& \quad=\Delta a_{\mu}^{t t b}(2 \text { loop }-\mathrm{BZ})\left(m_{t} \rightarrow m_{T}, N_{t}^{c} \rightarrow N_{T}^{c}\right)
\end{aligned}
$$

where for the vector-like fermion, $N_{T}^{c}=1$.

By the way, we should note that in the Barr-Zee 2-loop diagrams there are no two scalars or two $W^{ \pm}$charged bosons connect to the triangle loop simultaneously, which is induced 
by the helicity constraints since between the two charged particles, the fermion is the bottom qurak, which mass is much smaller than that of the top quark, and the slash momentum terms must vanish undergoing a single $\gamma$ matrix. Of course, the discussion here is very crude, and explicit and detailed discussion can be found in Ref. [64].

As the enhancement factor $m_{f}^{2} / m_{\mu}^{2}$ could easily overcome the loop suppression phase space factor $\alpha / \pi$, the two-loop contributions can be larger than one-loop ones. In the LRTH, since the CP-odd Higgs coupling to the lepton is proportional to $m_{v_{R}}$, the LRTH can sizably enhance the muon $g-2$ for a light CP-odd scalar and a large right-handed neutrino mass $m_{v_{R}}$.

\section{Global fit of the $125 \mathrm{GeV}$ Higgs}

The $125 \mathrm{GeV}$ Higgs signal data include a large number of observables and we will perform a global fit to the $125 \mathrm{GeV}$ Higgs signal data.

For the given neutral SM-like scalar-field $h$ and its couplings, the $\chi_{h}^{2}$ function can be defined as

$\chi_{h}^{2}=\sum_{k} \frac{\left(\mu_{k}-\hat{\mu}_{k}\right)^{2}}{\sigma_{k}^{2}}$,

where $k$ runs over the different production(decay) channels considered, and $\mu_{k}$ is the corresponding theoretical predictions for the LRTH parameters, as given later in Eqs. (34) and (40). $\hat{\mu}_{k}$ and $\sigma_{k}$ denote the measured Higgs signal strengths and their one-sigma errors, respectively, and their choices in this work appear in [65-75], though the data and the references listed are not complete.

\subsection{Relevant Lagrangian and the couplings}

After diagonaling, the Yukawa lagrangians can be written as,

$$
\mathcal{L}_{Y}=-\sum_{f=d, l} y_{f} h \bar{f} P_{R} f-\sum_{f=t, T} y_{f} h \bar{u} P_{L} u .
$$

From Eq. (28) and the couplings in Ref. [17], we can get the interactions between the Higgs boson and the pairs of $\bar{b} b, \bar{l} l, t \bar{t}, T \bar{T}, V V\left(V=W, W_{H}\right), \phi^{+} \phi^{-}$:

$$
\begin{gathered}
y_{b}=-\frac{m_{b}}{v} C_{L} C_{R}=-\frac{m_{b}}{v} \rho_{b}, y_{l}=-\frac{m_{l}}{v} C_{L} C_{R}=-\frac{m_{l}}{v} \rho_{l}, \\
y_{t}=-\frac{m_{t}}{v} C_{L} C_{R}=-\frac{m_{t}}{v} \rho_{t}, y_{T}=y\left(S_{R} S_{L}-C_{L} C_{R} x\right) / \sqrt{2},
\end{gathered}
$$

$h W_{\mu}^{+} W_{v}^{-}: \quad e m_{W} / s_{W}=\rho_{W} m_{W}^{2} / v, \quad \rho_{W}=e v /\left(m_{w} s_{W}\right)$ $h W_{H \mu}^{+} W_{H \nu}^{-}:-e^{2} f \times g_{\mu \nu} /\left(\sqrt{2} s_{w}^{2}\right)=y_{W_{H}} g_{\mu \nu}$,

$h \phi^{+} \phi^{-}: y_{\phi}, y_{\phi}=-x \frac{2 m_{h}^{2}-2 m_{\phi}^{2}}{3 \sqrt{2} f}$.

$\rho_{t}=\rho_{b}=\rho_{\tau}=C_{L} C_{R}, \rho_{W}=\frac{e * v}{m_{W} S_{W}}$ are the ratios of $h f f, h W W$ vertexes in LRTH and the standard models.

\subsection{Higgs signal strengths}

The so-called signal strengths, which are employed in the experimental data on Higgs searches, measuring the observable cross sections in ratio to the corresponding SM predictions. At the LHC, the SM-like Higgs particle is generated by the following relevant production mechanisms: gluon fusion $(g g \rightarrow H)$, vector boson fusion $\left(q q^{\prime} \rightarrow\right.$ $q q^{\prime} V V \rightarrow q q^{\prime} H$ ), associated production with a vector boson $\left(q \bar{q}^{\prime} \rightarrow W H / Z H\right)$, and the associated production with a $t \bar{t}$ pair $(q \bar{q} / g g \rightarrow t \bar{t} H)$. The Higgs decay channels are $\gamma \gamma$, $Z Z^{(*)}, W W^{(*)}, b \bar{b}$ and $\tau^{+} \tau^{-}$.

In order to fit the experimental measurements, we can write down the following ratios:

$$
\begin{aligned}
& \mu_{g g \gamma \gamma} \equiv \frac{\sigma(p p \rightarrow h) \operatorname{Br}(h \rightarrow \gamma \gamma)}{\sigma(p p \rightarrow H)_{\mathrm{SM}} \operatorname{Br}(H \rightarrow \gamma \gamma)_{\mathrm{SM}}} \\
& \mu_{t \bar{t} h \gamma \gamma} \equiv \frac{\sigma(p p \rightarrow t \bar{t} h) \operatorname{Br}(h \rightarrow \gamma \gamma)}{\sigma(p p \rightarrow t \bar{t} H)_{\mathrm{SM}} \operatorname{Br}(H \rightarrow \gamma \gamma)_{\mathrm{SM}}}, \\
& \mu_{g g V V} \equiv \frac{\sigma(p p \rightarrow h) \operatorname{Br}(h \rightarrow V V)}{\sigma(p p \rightarrow H)_{\mathrm{SM}} \operatorname{Br}(H \rightarrow V V)_{\mathrm{SM}}}, \\
& \mu_{t \bar{t} h V V} \equiv \frac{\sigma(p p \rightarrow t \bar{t} h) \operatorname{Br}(h \rightarrow V V)}{\sigma(p p \rightarrow t \bar{t} H)_{\mathrm{SM}} \operatorname{Br}(H \rightarrow V V)_{\mathrm{SM}}}, \\
& \mu_{g g f f} \equiv \frac{\sigma(p p \rightarrow h) \operatorname{Br}(h \rightarrow f f)}{\sigma(p p \rightarrow H)_{\mathrm{SM}} \operatorname{Br}(H \rightarrow f f)_{\mathrm{SM}}}, \\
& \mu_{t \bar{t} h f f} \equiv \frac{\sigma(p p \rightarrow t \bar{t} h) \operatorname{Br}(h \rightarrow f f)}{\sigma(p p \rightarrow t \bar{t} H)_{\mathrm{SM}} \operatorname{Br}(H \rightarrow f f)_{\mathrm{SM}}}, \\
& \mu_{V h \gamma \gamma} \equiv \frac{\sigma(p p \rightarrow V h) \operatorname{Br}(h \rightarrow \gamma \gamma)}{\sigma(p p \rightarrow V H)_{\mathrm{SM}} \operatorname{Br}(H \rightarrow \gamma \gamma)_{\mathrm{SM}}}, \\
& \mu_{V B F \gamma \gamma} \equiv \frac{\sigma(p p \rightarrow q q h) \operatorname{Br}(h \rightarrow \gamma \gamma)}{\sigma(p p \rightarrow q q H)_{\mathrm{SM}} \operatorname{Br}(H \rightarrow \gamma \gamma)_{\mathrm{SM}}}, \\
& \mu_{V h V V} \equiv \frac{\sigma(p p \rightarrow V h) \operatorname{Br}(h \rightarrow V V)}{\sigma(p p \rightarrow H)_{\mathrm{SM}} \operatorname{Br}(H \rightarrow V V)_{\mathrm{SM}}}, \\
& \mu_{V B F V V} \equiv \frac{\sigma(p p \rightarrow q q h) \mathrm{Br}(h \rightarrow V V)}{\sigma(p p \rightarrow q q H)_{\mathrm{SM}} \operatorname{Br}(H \rightarrow V V)_{\mathrm{SM}}}, \\
& \mu_{V h f f} \equiv \frac{\sigma(p p \rightarrow V h) \operatorname{Br}(h \rightarrow f f)}{\sigma(p p \rightarrow H)_{\mathrm{SM}} \operatorname{Br}(H \rightarrow f f)_{\mathrm{SM}}}, \\
& \mu_{V B F f f} \equiv \frac{\sigma(p p \rightarrow q q h) \operatorname{Br}(h \rightarrow f f)}{\sigma(p p \rightarrow q q H)_{\mathrm{SM}} \operatorname{Br}(H \rightarrow f f)_{\mathrm{SM}}},
\end{aligned}
$$

where $V=W, Z$. 

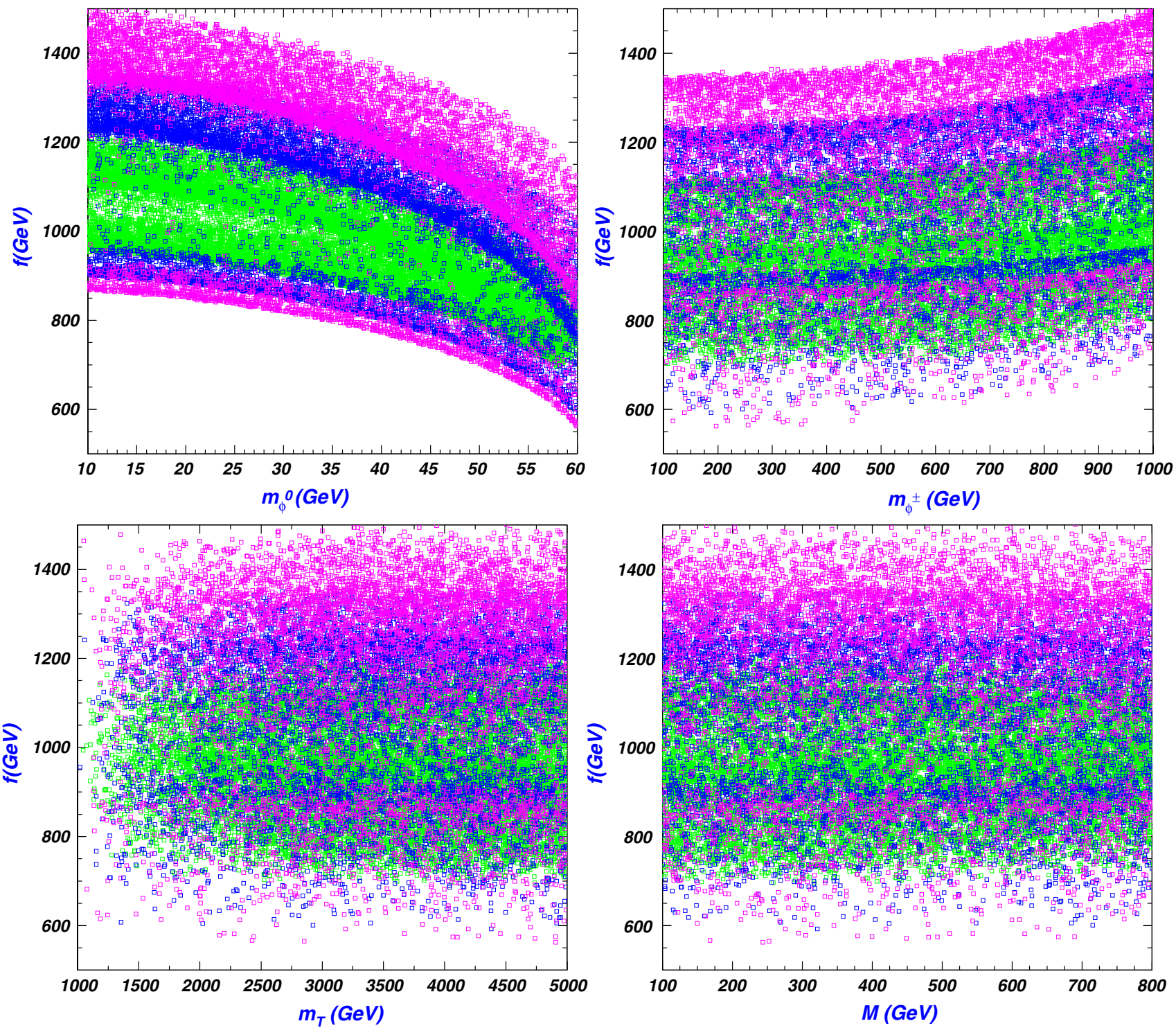

Fig. 3 The surviving samples within $1 \sigma, 2 \sigma$, and $3 \sigma$ ranges of $\chi_{h}^{2}$ on the planes of $f$ versus $m_{\phi^{0}}, m_{\phi^{ \pm}}, m_{T}$, and $M$. The green, blue and the pink points are respectively within the $1 \sigma, 2 \sigma$, and $3 \sigma$ regions of $\chi_{h}^{2}$

The ratio of the branching fraction will be expressed as:

$\frac{\operatorname{Br}(h \rightarrow X)}{\operatorname{Br}(H \rightarrow X)_{\mathrm{SM}}}=\frac{1}{\rho(h)} \frac{\Gamma(h \rightarrow X)}{\Gamma(H \rightarrow X)_{\mathrm{SM}}}$,

where $\rho(h)$ is the total decay width of the scalar $h$ in units of the SM Higgs width,

$$
\begin{aligned}
\rho(h) & =\frac{\Gamma(h)}{\Gamma_{\mathrm{SM}}(H)} \\
& =\frac{\Gamma^{B S M}(h)+\Gamma\left(h \rightarrow \varphi^{0} \varphi^{0}\right)}{\Gamma_{\mathrm{SM}}(H)} \\
& =\frac{\Gamma^{B S M}(h)}{\Gamma_{\mathrm{SM}}(H)}+\frac{\Gamma\left(h \rightarrow \varphi^{0} \varphi^{0}\right)}{\Gamma_{\mathrm{SM}}(H)},
\end{aligned}
$$

where the existence of the $h \rightarrow \varphi^{0} \varphi^{0}$ means in the LRTH models when $\varphi^{0}$ mass is less than $m_{h} / 2$, the channel $h \rightarrow \varphi^{0} \varphi^{0}$ will be open, and the total width of $h$ should changed into $\Gamma^{L R T H}(h)+\Gamma\left(h \rightarrow \varphi^{0} \varphi^{0}\right)$, where $\Gamma^{L R T H}(h)$ is corresponding to $\mathrm{SM}$ channels. $\Gamma\left(h \rightarrow \varphi^{0} \varphi^{0}\right)$ can be written as

$\Gamma\left(h \rightarrow \varphi^{0} \varphi^{0}\right)=\frac{g_{h \varphi^{0} \varphi^{0}}^{2}}{8 \pi m_{h}} \sqrt{1-\frac{4 m_{\varphi}^{2}}{m_{h}^{2}}}$

where $g_{h \varphi^{0} \varphi^{0}}=\frac{v m_{h}^{2}}{54 f^{2}}\left[11+15\left(1-\frac{2 m_{\varphi}^{2}}{m_{h}^{2}}\right)\right]$ [77].

Particularizing to the LRTH and assuming only one dominant production channel in each case, we have: 

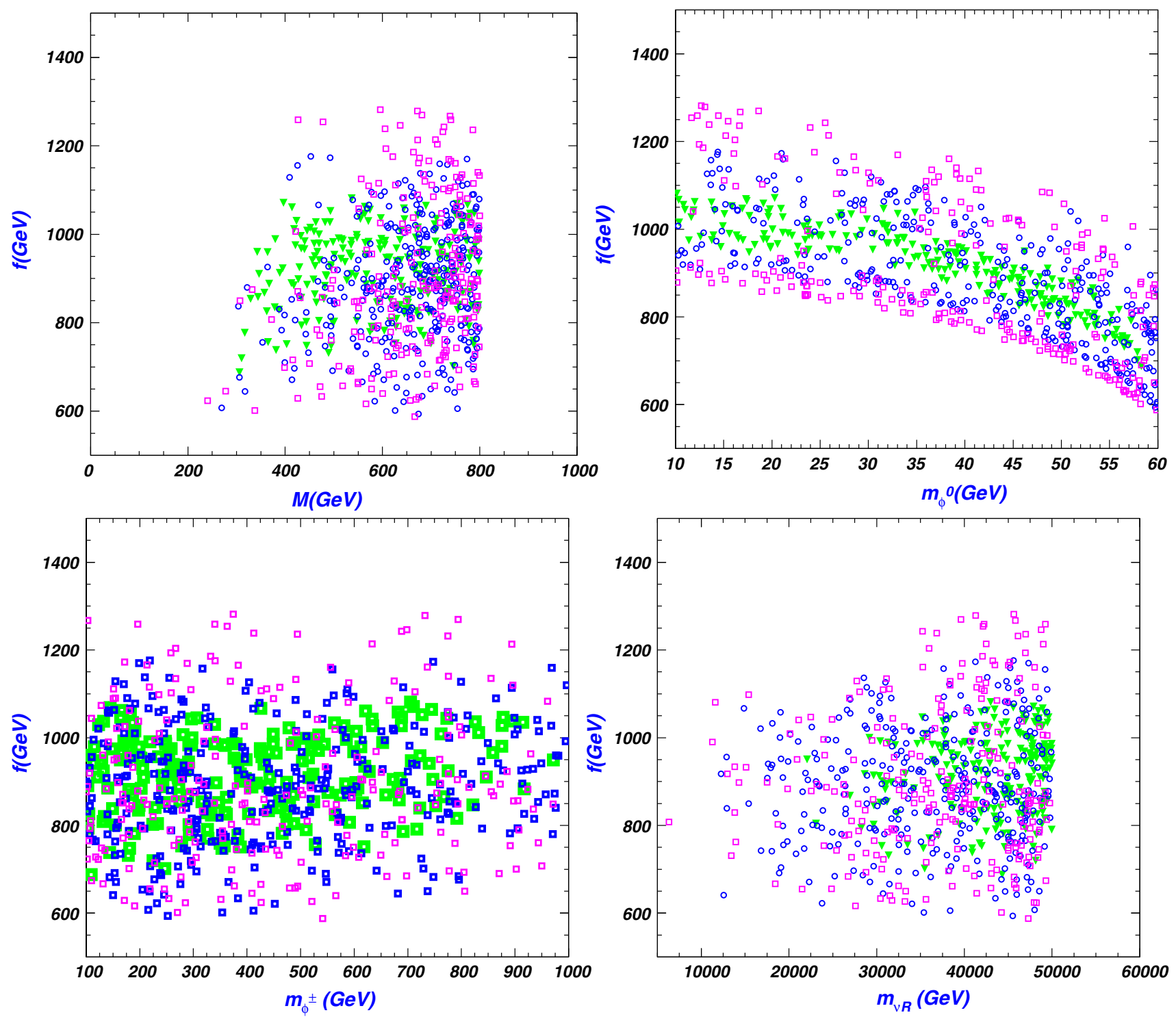

Fig. 4 The samples satisfying the constraints of Higgs global fit $\chi_{h}^{2}$ within $1 \sigma, 2 \sigma$, and $3 \sigma$ (green, blue and the pink points respectively) ranges, on the planes of $f$ versus $M, m_{\phi^{0}}, m_{\phi^{ \pm}}$, and $m_{v_{R}}$, with the

constraints of the $a_{\mu}$ from the experiemnts. All the samples are allowed by the constraints of muon $g-2$, which is given in Eq. (3)

$\mu_{g g \gamma \gamma}=C_{g g} C_{\gamma \gamma} \rho(h)^{-1}, \mu_{g g V V}=C_{g g} \rho_{W}^{2} \rho(h)^{-1}$,

$\mu_{g g f f}=C_{g g} \rho_{f}^{2} \rho(h)^{-1}, \mu_{t \bar{t} h \gamma}=\rho_{t}^{2} C_{\gamma \gamma} \rho(h)^{-1}$,

$\mu_{t \bar{t} h V V}=\rho_{t}^{2} \rho_{W}^{2} \rho(h)^{-1}, \mu_{t \bar{t} h f f}=\rho_{t}^{2} \rho_{f} \rho(h)^{-1}$,

$\mu_{V B F \gamma \gamma}=\rho_{W}^{2} C_{\gamma \gamma} \rho(h)^{-1}, \mu_{V B F V V}=\rho_{W}^{2} \rho_{W}^{2} \rho(h)^{-1}$,

$\mu_{V B F f f}=\rho_{W}^{2} \rho_{f}^{2} \rho(h)^{-1}, \mu_{V h \gamma \gamma}=\rho_{W}^{2} C_{\gamma \gamma} \rho(h)^{-1}$,

$\mu_{V h V V}=\rho_{W}^{2} \rho_{W}^{2} \rho(h)^{-1}, \mu_{V h f f}=\rho_{W}^{2} \rho_{f} \rho(h)^{-1}$.

Note that $\rho_{W}=\rho_{Z}$.

The one-loop functions are given by

$C_{g g}=\frac{\sigma(g g \rightarrow h)}{\sigma(g g \rightarrow h)_{\mathrm{SM}}}=\frac{\left|\sum_{q=t, T} y_{q} \mathcal{F}\left(x_{q}\right)\right|^{2}}{\left|\sum_{q=t} y_{t} \mathcal{F}\left(x_{q}\right)\right|^{2}}$

where $y_{t}=y_{t} v / \sqrt{2}$, and

$$
\begin{aligned}
C_{\gamma \gamma} & =\frac{\Gamma(h \rightarrow \gamma \gamma)}{\Gamma(h \rightarrow \gamma \gamma)_{\mathrm{SM}}} \\
& =\frac{\left|\sum_{f} y_{f} N_{C}^{f} Q_{f}^{2} \mathcal{F}\left(x_{f}\right)+\mathcal{F}_{1}\left(x_{W}\right) y_{W}+\mathcal{F}_{1}\left(x_{W_{H}}\right) y_{W_{H}}+\mathcal{F}_{0}\left(x_{\phi^{ \pm}}\right) y_{\phi}\right|^{2}}{\left|\sum_{f} N_{C}^{f} Q_{f}^{2} \mathcal{F}\left(x_{f}\right)+\mathcal{G}\left(x_{W}\right)\right|^{2}}
\end{aligned}
$$

with $N_{C}^{f}$ and $Q_{f}$ the number of colours and the electric charge of the fermion $f$, and $x_{f}=4 m_{f}^{2} / M_{h}^{2}, x_{W}=$ $4 M_{W}^{2} / M_{h}^{2}$ and $x_{\phi^{ \pm}}=4 M_{\phi^{ \pm}}^{2} / M_{h}^{2}$. Note that the ratios (34) are defined for $M_{h}=M_{h_{\mathrm{SM}}}$. The functions $\mathcal{F}\left(x_{f}\right)$ and $\mathcal{F}_{1}\left(x_{W}\right)$ contain the contributions of the triangular 1-loop 
from fermions and $W^{ \pm}$bosons. The masses of the first two fermion generations will be neglected. Since $\mathcal{F}\left(x_{f}\right)$ vanishes for massless fermions, we only need to consider the top and the vector-like top contributions, which correspond large Yukawa couplings.

The explicit expressions of the different loop functions can be given as:

$$
\begin{aligned}
\mathcal{F}(x) & =\frac{x}{2}[4+(x-1) f(x)], \\
\mathcal{F}_{1}(x) & =-2-3 x+\left(\frac{3}{2} x-\frac{3}{4} x^{2}\right) f(x), \\
\mathcal{F}_{0}(x) & =-x-\frac{x^{2}}{4} f(x), \\
\mathcal{K}(x) & =-\frac{x}{2} f(x),
\end{aligned}
$$

with

$$
f(x)=\left\{\begin{array}{ll}
-4 \arcsin ^{2}(1 / \sqrt{x}), & x \geqslant 1 \\
{\left[\ln \left(\frac{1+\sqrt{1-x}}{1-\sqrt{1-x}}\right)-i \pi\right]^{2},} & x<1
\end{array} .\right.
$$

\section{Results and discussions}

In Fig. 3, we project the surviving samples within $1 \sigma, 2 \sigma$, and $3 \sigma$ ranges of $\chi_{h}^{2}$ on the planes of $f$ versus $m_{\phi^{0}}, m_{\phi^{ \pm}}, m_{T}$, and $M$, the exclusion limits from searches for Higgs at LEP, the signal data of the $125 \mathrm{GeV}$ Higgs, and the flavor changing constraints of $\mu \rightarrow e \gamma[18,19]$. Figure 3 shows that the of $\chi_{h}^{2}$ value favors a bit large $f$. We can see from the upper panel that if the value of $f$ is small, the value of $\chi_{h}^{2}$ prefer to have a large $m_{\phi^{0}}$ and a small $m_{\phi^{ \pm}}$. From the lower-left panel of Fig. 3 that the value of $\chi_{h}^{2}$ is favored to a large top partner mass $m_{T}$.

In Fig. 4, we project the surviving samples within $1 \sigma, 2 \sigma$, and $3 \sigma$ on the planes of $f$ versus $m_{\phi^{0}}, m_{\phi^{ \pm}}, m_{T}$, and $M$ after imposing the constraints from the muon $g-2$ anomaly, the lepton flavor changing decay. The upper-left panel shows that the surviving data preferring to a large mixing parameter $M$, about $200-800 \mathrm{GeV}$.

Figure 4 shows that with the limits from muon $g-2$, the Higgs global fit and the lepton decay $\mu \rightarrow e \gamma$ being satisfied, the muon $g-2$ anomaly can be explained in the regions of 200 $\mathrm{GeV} \leq M \leq 800 \mathrm{GeV}, 700 \mathrm{GeV} \leq f \leq 1100 \mathrm{GeV}, 10 \mathrm{GeV}$ $\leq m_{\phi^{0}} \leq 60 \mathrm{GeV}, 100 \mathrm{GeV} \leq m_{\phi^{ \pm}} \leq 900 \mathrm{GeV}$, and $m_{v_{R}} \geq$ $15 \mathrm{TeV}$. Figure 4 shows that in the range of $10 \mathrm{GeV} \leq m_{\phi^{0}} \leq$ $60 \mathrm{GeV}$ and a light $f$ constrained by the decay $\mu \rightarrow e \gamma$, the muon $g-2$ anomaly can be explained for a large enough $m_{v_{R}}$, which constraint severely the models which introduce extra right-handed neutrinos to give the natural light neutrino masses. Since the contributions of $m_{v_{R}}$ to the muon $g-2$ anomaly have destructive interference with the prediction, there may not exist many samples, and the model survives in narrow space, as shown in the lower-right panel of Fig. 4.

\section{Conclusion}

The muon $g-2$ anomaly can be explained in the LRTH model. After imposing various relevant theoretical and experimental constraints, we performed a scan over the parameter space of this model to identify the ranges in favor of the muon $g-2$ explanation, and the Higgs direct search limits from LHC constraint strongly. We find that the muon $g-2$ anomaly can be accommodated in the region of $200 \mathrm{GeV}$ $\leq M \leq 800 \mathrm{GeV}, 700 \mathrm{GeV} \leq f \leq 1100 \mathrm{GeV}, 10 \mathrm{GeV}$ $\leq m_{\phi^{0}} \leq 60 \mathrm{GeV}, 100 \mathrm{GeV} \leq m_{\phi^{ \pm}} \leq 900 \mathrm{GeV}$, and $m_{v_{R}} \geq$ $15 \mathrm{TeV}$, after imposing the joint constraints from the theory, the precision electroweak data, the $125 \mathrm{GeV}$ Higgs signal data, and the leptonic decay.

Acknowledgements The author would appreciate the helpful discussions with Fei Wang, Wen-Yu Wang and Lei Wang. This work was supported by the National Natural Science Foundation of China under Grants 11675147,11605110 and by the Academic Improvement Project of Zhengzhou University.

Data Availability Statement This manuscript has associated data in a data repository. [Authors' comment: All data generated or analysed during this study are included in this published article (and its supplementary information files).]

Open Access This article is distributed under the terms of the Creative Commons Attribution 4.0 International License (http://creativecomm ons.org/licenses/by/4.0/), which permits unrestricted use, distribution, and reproduction in any medium, provided you give appropriate credit to the original author(s) and the source, provide a link to the Creative Commons license, and indicate if changes were made.

Funded by SCOAP $^{3}$.

\section{References}

1. H.N. Brown et al. [Muon g-2 Collaboration], Phys. Rev. Lett. 86, 2227 (2001). arXiv:hep-ex/0102017

2. G.W. Bennett et al. [Muon g-2 Collaboration], Phys. Rev. D 73, 072003 (2006). arXiv:hep-ex/0602035

3. G.W. Bennett et al. (Muon g-2), Phys. Rev. D 73, 072003 (2006). arXiv:hep-ex/0602035

4. C. Patrignani et al. (Particle Data Group), Chin. Phys. C 40, 100001 (2016)

5. J.P. Miller, E. de Rafael, B.L. Roberts, Rep. Prog. Phys. 70, 795 (2007). arXiv:hep-ph/0703049

6. F. Jegerlehner, A. Nyffeler, Phys. Rep. 477, 1 (2009). arXiv:0902.3360

7. J.P. Miller, E. de Rafael, B.L. Roberts, D. Stockinger, Ann. Rev. Nucl. Part. Sci. 62, 237 (2012)

8. M. Lindner, M. Platscher, F.S. Queiroz, Phys. Rep. 731, 1 (2018). arXiv: 1610.06587

9. F. Jegerlehner, Springer Tracts Mod. Phys. 274, 1 (2017)

10. Z. Chacko, H.-S. Goh, R. Harnik, Phys. Rev. Lett. 96, 231802 (2006). arXiv:hep-ph/0506256 
11. Z. Chacko, Y. Nomura, M. Papucci, G. Perez, JHEP 0601, 126 (2006). arXiv:hep-ph/0510273

12. Z. Chacko, H. Goh, R. Harnik, JHEP 0601, 108 (2006). arXiv:hep-ph/0512088

13. J.C. Pati, A. Salam, Phys. Rev. D 10, 275 (1974)

14. R.N. Mohapatra, J.C. Pati, Phys. Rev. D 11, 566 (1975)

15. R.N. Mohapatra, J.C. Pati, Phys. Rev. D 11, 2558 (1975)

16. H.S. Goh, C. Krenke, Phys. Rev. D 76, 115018 (2007). arXiv:0707.3650

17. H.-S. Goh, S. Su, Phys. Rev. D 75, 075010 (2007). arXiv:hep-ph/0611015

18. A. Abada, I. Hidalgo, Phys. Rev. D 77, 113013 (2008). arXiv:hep-ph/0711.1238

19. G.-L. Liu, F. Wang, K. Xie, X.-F. Guo, Phys. Rev. D 96, 035005 (2017). arXiv:1701.00947

20. Particle Data Group, W.-M. Yao et al., J. Phys. G 33, 1 (2006)

21. M.E.G. Collaboration, J. Adam et al., Phys. Rev. Lett. 110, 201801 (2013). arXiv:1303.0754

22. MEG Collaboration, A.M. Baldini et al., Eur. Phys. J. C 76(8), 434 (2016). arXiv:1605.05081

23. H.-S. Goh, C.A. Krenke, Phys. Rev. D 81, 055008 (2010). arXiv:0911.5567

24. Y.-B. Liu, Z.-J. Xiao, J. Phys. G 42, 065005 (2015). arXiv: 1412.5905

25. C.-X. Yue, H.-D. Yang, W. Ma, Nucl. Phys. B 818, 1 (2009). arXiv:0903.3720

26. L. Wang, J.M. Yang, JHEP 1005, 024 (2010). arXiv: 1003.4492

27. G. Zhan-Ying, Y. Guang, Y. Bing-Fang, Chin. Phys. C 37, 103101 (2013). arXiv:1304.2249

28. Y.-B. Liu, Z.-J. Xiao, Nucl. Phys. B 892, 63 (2015). arXiv: 1409.6050

29. E.M. Dolle, S. Shufang, Phys. Rev. D 77, 075013 (2008). arXiv:0712.1234

30. J.-Y. Lee, D.-W. Jung, J. Korean Phys. Soc. 63, 1114 (2013). hep$\mathrm{ph} / 0701071$

31. M.C. Gonzalez-Garcia, Y. Nir, Rev. Mod. Phys. 75, 345 (2003)

32. V. Barger, D. Marfatia, K. Whisnant, Int. J. Mod. Phys. E 12, 569 (2003)

33. A.Y. Smimov, Talk given at the 2nd International Workshop on Neutrino oscillations in Venice (NOVE) December 3-5 (Venice, Italy, 2003). arXiv:hep-ph/0402264

34. M.C. Gonzalez-Garcia, M. Maltoni, Phys. Rep. 460, 1 (2007). arXiv:0704.1800

35. L. Wang, J.M. Yang, M. Zhang, Y. Zhang, Phys. Lett. B 788, 519 (2019). arXiv: 1809.05857

36. G. Aad et al. (ATLAS Collaboration), JHEP 1211, 094 (2012)

37. G. Aad et al., Phys. Lett. B 712, 22 (2012)

38. G. Aad et al., Phys. Rev. D 86, 012007 (2012)

39. S. Chatrchyan et al. (CMS Collaboration), JHEP 1301, 154 (2013)

40. S. Chatrchyan et al., Phys. Lett. B 718, 307 (2012)

41. T. Aaltonen et al. (CDF Collabration), Phys. Rev. Lett. 102, 031801 (2009)

42. V.M. Abazov et al. (D0 Collaboration), Phys. Lett. B 695, 88 (2011)

43. J. Beringer et al. (Particle Data Group collaboration), Phys. Rev. D 86, 010001 (2012)

44. S. Chatrchyan et al. (CMS Collaboration), Phys. Lett. B 720, 63 (2013)

45. Y.-B. Liu, S. Cheng, Z.-J. Xiao, Phys. Rev. D 89, 015013 (2014)

46. G. Aad et al. (ATLAS Collaboration), Phys. Lett. B 718, 1284 (2013)

47. J.P. Leveille, Nucl. Phys. B 137, 63 (1978)
48. S.R. Moore, K. Whisnant, B.-L. Young, Phys. Rev. D 31, 105 (1985)

49. F.S. Queiroz, W. Shepherd, Phys. Rev. D 89, 095024 (2014) arXiv:1403.2309

50. G.-L. Liu, F. Wang, S. Yang, Phys. Rev. D 88, 115006 (2013). arXiv: 1302.1840

51. A. Broggio, E.J. Chun, M. Passera, K.M. Patel, S.K. Vempati, JHEP 1411, 058 (2014). arXiv: 1409.3199 [hep-ph]

52. L. Wang, X.F. Han, JHEP 05, 039 (2015). arXiv:1412.4874 [hep$\mathrm{ph}]$

53. A. Dedes, H.E. Haber, JHEP 0105, 006 (2001). hep-ph/0102297

54. J.F. Gunion, JHEP 0908, 032 (2009). arXiv:0808.2509 [hep-ph]

55. K.M. Cheung, C.H. Chou, O.C.W. Kong, Phys. Rev. D 64, 111301 (2001). hep-ph/0103183

56. D. Chang, W.F. Chang, C.H. Chou, W.Y. Keung, Phys. Rev. D 63, 091301 (2001). arXiv:hep-ph/0009292

57. M. Krawczyk, Acta Phys. Polon. B 33, 2621 (2002). hep$\mathrm{ph} / 0208076$

58. F. Larios, G. Tavares-Velasco, C.P. Yuan, Phys. Rev. D 64, 055004 (2001). hep-ph/0103292

59. K. Cheung, O.C.W. Kong, Phys. Rev. D 68, 053003 (2003). hep$\mathrm{ph} / 0302111$

60. A. Arhrib, S. Baek, Phys. Rev. D 65, 075002 (2002). hep$\mathrm{ph} / 0104225$

61. S. Heinemeyer, D. Stockinger, G. Weiglein, Nucl. Phys. B 690, 62 (2004). hep-ph/0312264

62. O.C.W. Kong, talk at SUSY03, Report number: NCU-HEP-k011. arXiv:hep-ph/0402010

63. K. Cheung, O.C.W. Kong, J.S. Lee, JHEP 0906, 020 (2009). arXiv:0904.4352 [hep-ph]

64. V. Ilisie, JHEP 04, 077 (2015). arXiv:1502.04199 [hep-ph]

65. ATLAS Collaboration, Phys. Rev. D 98, 052003 (2018). arXiv: 1807.08639

66. C.M.S. Collaboration, Phys. Lett. B 780, 501 (2018). arXiv: 1709.07497 [hep-ex]

67. C.M.S. Collaboration, JHEP 03, 026 (2019). arXiv:1804.03682 [hep-ex]

68. ATLAS Collaboration, Phys. Lett. B 789, 508 (2019). arXiv: 1808.09054

69. C.M.S. Collaboration, JHEP 08, 066 (2018). arXiv:1803.05485 [hep-ex]

70. C.M.S. Collaboration, JHEP 05, 104 (2014), arXiv:1401.5041 [hep-ex]

71. C.M.S. Collaboration, JHEP 11, 047 (2017). arXiv:1706.09936 [hep-ex]

72. C.M.S. Collaboration, JHEP 11, 185 (2018). arXiv:1804.02716 [hep-ex]

73. C.M.S. Collaboration, JHEP 11, 152 (2018). arXiv:1806.05996 [hep-ex]

74. CMS Collaboration, Search for the standard model Higgs boson decaying into two muons in pp collisions at $\mathrm{sqrt}(\mathrm{s})=13 \mathrm{TeV}$, Tech. Rep. CMS-PAS-HIG-17-019, CERN, Geneva (2017)

75. ATLAS Collaboration, Phys. Rev. Lett. 119, 051802 (2017). arXiv:1705.04582 [hep-ex]

76. A. Crivellin, J. Heeck, P. Stoffer, Phys. Rev. Lett. 116, 081801 (2016). arXiv: 1507.07567

77. Y.-B. Liu, Z.-J. Xiao, JHEP 1402, 128 (2014). arXiv:1312.4004 